\title{
DRIVING SIMULATOR PERFORMANCE IN THE ACUTE POST-INJURY PHASE FOLLOWING A MILD TRAUMATIC BRAIN INJURY AMONG YOUNG DRIVERS
}

\author{
Despina Stavrinos ${ }^{1}$, Ginger Yang ${ }^{2}$, Thomas Kerwin ${ }^{3}$, Benjamin McManus ${ }^{1}$, \\ Tyler R. Bell ${ }^{1}$, Alison Newton ${ }^{2}$, Bhavna Singichetti ${ }^{2}$ \\ ${ }^{1}$ University of Alabama at Birmingham, Birmingham, AL, USA \\ ${ }^{2}$ Nationwide Children's Hospital, Center for Injury Research and Policy, Columbus, OH, USA \\ ${ }^{3}$ The Ohio State University, Columbus, OH, USA \\ Email: dstavrin@uab.edu
}

\begin{abstract}
Summary: While mild traumatic brain injury (mTBI) can lead to cognitive and functional impairments, little is known about how mTBI may affect driving, especially among young drivers who are at an increased risk of mTBI and motor vehicle collisions compared to other age groups. The objective of this multisite, pilot study was to examine the feasibility of assessing driving performance acutely post-injury (i.e., $\mathrm{mTBI}$ sustained $<2$ weeks at assessment) among young drivers with and without $\mathrm{mTBIs}\left(\mathrm{N}=42 ; \mathrm{n}_{\mathrm{mTBI}}=21 ; \mathrm{n}_{\text {control }}=21\right)$ using high-fidelity driving simulators. Driving performance was hypothesized to be significantly degraded, especially under conditions of high cognitive load, among drivers with mTBI compared to matched controls. Neurocognitive measures used in clinical assessment of mTBI (i.e., Cogstate Brief Battery) were hypothesized to correlate with driving simulator performance metrics. Risk management protocols were successful (i.e., no participants withdrew due to simulator sickness) and no significant increase in post-concussion symptoms was found from pre-assessment to immediately following driving assessment. Group differences on key driving variables did not emerge; however, drivers with mTBI showed a differential pattern of driving under high cognitive load. Neurocognitive correlates of simulated driving performance suggested processing speed, attention, and working memory are important functions for driving. Implications and future directions discussed.
\end{abstract}

\section{INTRODUCTION}

Resuming driving after a mild traumatic brain injury (mTBI) is frequently an immediate goal for patients in resuming their daily activities (Preece, Geffen, \& Horswill, 2013). While physical and cognitive rest are commonly prescribed for mTBI patients in the US, physicians have little guidance to help determine when it is safe for their patients to resume driving. Currently, no specific guidelines exist in the US on driving after a mTBI (McCrory et al., 2013). Clinical guidelines in Canada and Australia recommend "no driving within 24 hours of a mTBI," but are based solely on expert consensus (Marshall, Bayley, McCullagh, Velikonja, \& Berrigan, 2012). Young drivers are of particular concern because they have the highest rates of mTBI of all age groups (Centers for Disease Control and Prevention, 2016; McKinlay et al., 2008) as well as the highest crash rates of all drivers (Insurance Institute for Highway Safety [IIHS], 2013; Williams, 2003). Given the significant reduction in mental resources available for performing driving tasks following an mTBI (Grady, Master, \& Gioia, 2012; Strayer et al., 2015), comprehensive research is urgently needed to determine the effects of mTBI on driving performance.

Driving is a complex task requiring motor coordination, visual perception, and higher-order cognition (e.g., attention, planning, decision making, monitoring of behavior). Deficits in neurocognitive processing speed after mTBI may further exacerbate driving decrements. Individuals with 
mTBI may have difficulty locating, identifying, and reacting to stimuli (Collie, Darby, \& Maruff, 2001), as well as longer processing times for decision and non-decision processes (e.g., increased duration of sensory processing and/or response execution time) (Del Rossi, 2017; Ratcliff \& McKoon, 2008). Existing evidence shows that adults with mTBI exhibit significantly slower responses in identifying hazardous traffic situations and take longer to complete driving-related tasks compared to orthopedic injury controls (Baker, Unsworth, \& Lannin, 2015; Preece, Horswill, \& Geffen, 2010). These deficits may be amplified in young drivers with less developed driving skills. After mTBI, fewer mental resources are available to perform complex tasks such as driving (Cossette, Ouellet, \& McFadyen, 2014; Strayer et al., 2013). Young drivers have yet to internalize basic driving tasks making them more vulnerable to interference by other cognitive demands (McCartt, Shabanova, \& Leaf, 2003). Thus, mTBI may have a greater effect on young drivers' performance compared to that of adults when neurocognitive function is impaired.

This pilot study was among the first to test the feasibility of assessing driving performance among young drivers with mTBI using a high-fidelity driving simulator, particularly in the acute post-injury phase. Specific aims and associated hypotheses were: Aim 1: Examine the differences in simulated driving performance among young drivers with and without mTBI. Hypothesis: Drivers with mTBI will perform more poorly, acutely post-injury (increased total braking reaction time, speed variation, lane position variation). Aim 2: Determine whether increased cognitive load affects the driving performance of young drivers with mTBI more than controls. $\mathrm{Hy}$ pothesis: Performance of young drivers with $\mathrm{mTBI}$ in a driving simulator will be affected more than the performance of controls by increased cognitive load (mental resources required to perform a concurrent task while driving) (Strayer et al., 2013). Aim 3: Examine associations of driving performance variables with neurocognitive variables. Hypothesis: Poorer neuropsychological functioning will be significantly correlated with poorer driving simulator performance, especially under the most challenging condition (unexpected events and high cognitive load).

\section{METHOD}

\section{Overview}

The study utilized a prospective, repeated measures, 4 condition design to examine differences in driving performance among young drivers with and without $\mathrm{mTBI}\left(\mathrm{N}=42 ; \mathrm{n}_{\mathrm{mTBI}}=21 ; \mathrm{n}_{\text {control }}=21\right)$ using high-fidelity driving simulators at University of Alabama at Birmingham (UAB) and The Ohio State University (OSU). At both sites, participants were recruited from local concussion clinics and intramural/club sports teams. At OSU, study information was distributed during preseason team meetings. Inclusion criteria included: 1) being between the ages of 16-25 years; 2) identified and enrolled within 2 weeks of injury; and 3) having a valid driver's license and having driven in the past 12 months. Participants met criteria of mTBI by either physician confirmed diagnosis of concussion or by reporting substantial symptoms on the Post Concussive Symptom Scale (scores $\geq 13$ were eligible at screening). Individuals were excluded if their injury: 1 ) required surgery or hospital admission; 2) was motor vehicle collision-related; 3) was intentional or associated with illicit drug/alcohol use; or if they 5) lacked a valid driver license; 6) were not an active driver pre-injury; or 7) had comorbidities impacting fitness to drive (i.e., conditions affecting eyesight, dominant arm, or right leg). Healthy controls were matched on age, gender, driving experience (time since licensure), and type of licensure. Controls were recruited from a participant registry maintained in the UAB PI's lab and through community flyers. 


\section{Measures and Procedure}

Participant demographics (age, gender, race, months since licensure, type of licensure) and injury information (e.g., history of TBI, mTBI symptomology using the Post Concussive Symptom Scale for those in mTBI group) were collected. Participants were scheduled to complete an inperson lab assessment involving a driving simulator assessment and a neurocognitive battery.

Driving Simulator Assessment. Both sites had comparable driving simulators by Realtime Technologies (RTI) which included a full vehicle cab on a motion base (OSU: 6 DOF; UAB: 1 DOF) and front projection field of view of at least 180 degrees (OSU: 260 degrees). Following a brief (5 minute) acclimation period, participants completed four simulated 6 mile drives on a 4-lane divided freeway that included off-ramps, curves, and reactive ambient traffic. Clear, daytime weather and 65 miles-per-hour posted speed limits were constant across drives. Participants were instructed to drive as they normally would in a real car on a real road. A 2 (Event: no/yes) x 2 (Cognitive Load: no/yes) design was employed (both factors randomized across participants). Two drives featured two unexpected sudden on-screen events requiring evasive maneuvers. Two drives induced cognitive load by requiring participants to concurrently perform secondary tasks: engage in a naturalistic conversation with a passenger (trained staff member) for half the drive and perform an auditory version of the Operation Span (OSPAN) task for the other half. The order of concurrent tasks was randomized. Participants were advised to 1) inform staff if they experienced motion sickness, dizziness, or elevation of TBI symptoms, and 2) stop the assessment, as needed. Key driving performance variables (Table 1) were selected for their relation to potential mTBI-related cognitive impairments (Neyens, Boyle, \& Schultheis, 2015; Stavrinos et al., 2013). Braking reaction time was recorded for participants at UAB $(n=28)$.

Table 1. Key driving performance variables measured by driving simulators

\begin{tabular}{|c|c|}
\hline Driving Performance Variable & Operational Definition \\
\hline \multicolumn{2}{|c|}{ Continuously-Recorded Measures of Vehicle Control } \\
\hline Standard Deviation of Speed & $\begin{array}{l}\text { Fluctuation in driving speed with greater fluctuation indicating } \\
\text { inefficient driving }\end{array}$ \\
\hline Standard Deviation of Lane Position & $\begin{array}{l}\text { Standard measure of steering variability, which is a sensitive } \\
\text { measure of demand of secondary tasks }\end{array}$ \\
\hline Speed & Miles per hour driven \\
\hline \multicolumn{2}{|c|}{ Event-Only Recorded Measures of Driver Response } \\
\hline Braking Reaction Time & $\begin{array}{l}\text { Time between the presentation of stimulus and first force applied } \\
\text { to brake (sum of Neurological Time }+ \text { Foot Removal Time }+ \\
\text { Motion Time) }\end{array}$ \\
\hline
\end{tabular}

Neurocognitive Assessment. Participants completed a computerized neurocognitive assessment (Cogstate Brief Battery), consisting of four subtests assessing four cognitive domains: 1) Processing speed: The Detection Task measured psychomotor function via reaction time of detecting a target card facing up on the screen; 2) Attention: The Identification Task measured visual attention via reaction time and correctly selecting the key for each card; 3) Visual learning: The One Card Learning Task measured memory by testing whether a card was shown previously during the whole task; and 4) Working memory: The One Back Task measured working memory by testing whether the present card was the same as the single previous card.

Each subtest yielded an accuracy score (percent of correct trials) and a speed score (time to respond accurately, in milliseconds). The battery has excellent psychometric properties including test-retest reliability, and criterion and construct validity (Louey, et al., 2014). 


\section{Data Analysis}

Descriptive statistics were examined for key variables including demographics, mTBI characteristics, simulated driving performance, and neurocognitive functioning. Differences between drivers with mTBI and controls were compared using t-tests or Wilcoxon Mann-Whitney $U$ nonparametric tests. To examine the differences in simulated driving performance among drivers with and without mTBI, we analyzed and compared simulated driving performance across the 4 driving conditions. To determine whether increased cognitive load affected the driving performance of drivers with mTBI more than controls, we used Repeated Measures ANOVA to test the interaction "group X cognitive load." Differences in driving performance between the groups stratified by cognitive load level were illustrated if a significant interaction was found. To examine correlations of driving performance variables with neurocognitive outcomes, we calculated correlations of each of the 4 driving conditions with the neurocognitive variables for each group.

\section{RESULTS}

The study enrolled 48 participants across two study sites (UAB: $n=28$; OSU: $n=20$ ). Six participants ( $3 \mathrm{mTBI}$ cases and their 3 matched controls) were excluded from analyses due to PCSS scores $<13$ at screening. Table 2 provides participant characteristics for the 42 participants included in all analyses. Braking reaction time data were only collected at UAB $(n=28)$.

Table 2. Participant demographics and characteristics by group

\begin{tabular}{|c|c|c|c|c|c|c|}
\hline \multirow[t]{2}{*}{ Variable } & \multicolumn{3}{|c|}{ Drivers with mTBI $(n=21)$} & \multicolumn{3}{|c|}{ Controls $(n=21)$} \\
\hline & Mean $(\mathrm{SD})$ & $\mathrm{n}(\%)$ & Range & Mean (SD) & $\underline{\mathrm{n}(\%)}$ & Range \\
\hline Age (years) & $18.06(1.94)$ & & $16.13-23.64$ & $17.87(1.79)$ & & $16.12-22.95$ \\
\hline Gender (males) & & $8(36)$ & & & $7(35)$ & \\
\hline \multicolumn{7}{|l|}{ Race } \\
\hline White & & $18(82)$ & & & $15(75)$ & \\
\hline African American & & $2(9)$ & & & $4(20)$ & \\
\hline Asian & & $1(4.5)$ & & & $0(0)$ & \\
\hline More than one race & & $1(4.5)$ & & & $1(5)$ & \\
\hline Months since full licensure & $19.01(16.80)$ & & $1.77-67.52$ & $17.49(15.28)$ & & $2.10-67.29$ \\
\hline Driving assessment after injury (hours) & $212.73(101.09)$ & & $72.00-336.00$ & & & \\
\hline PCSS at screening & $34.33(17.28)$ & & $13.00-78.00$ & $15.50(21.35)$ & & $1.00-71.0$ \\
\hline PCSS at assessment & $30.00(20.09)$ & & $4.00-83.00$ & $13.80(15.13)$ & & $0.00-56.0$ \\
\hline
\end{tabular}

Differences between groups on neurocognitive variables revealed that drivers with mTBI exhibited marginally significant slower detection speed than controls $(U=321.00, p=.065)$.

\section{Primary Results}

No differences emerged between drivers with/without mTBI in any driving condition (Table 3). There were no significant injury group $X$ cognitive load interactions acutely post-injury (Table 4). For drivers with mTBI, driving speed was significantly higher in the cognitive load condition. For controls, standard deviation of lane position was significantly greater in the no load condition. For the mTBI group in the no load, no event (mundane) condition, worse performance in the neurocognitive domains of processing speed, working memory, and learning tasks was significantly associated with more lane position variability ( $r$ 's .46-.69). In the cognitive load, event present (demanding) condition, slower processing speed and worse attention was associated with more lane variability ( $r$ 's .41-.52). In the mundane condition, more speed variability was signifi- 
cantly associated with worse attention; whereas, in the demanding condition more speed variability was significantly associated with all four neurocognitive domains ( $r$ 's .43-.47). No significant correlations emerged between neurocognitive variables and speed or braking reaction time.

For controls in the mundane condition, worse performance on processing speed and working memory was significantly associated with more lane position variability ( $r$ 's .52-.53). In the demanding condition, worse performance on attention and working memory was significantly associated with more lane position variability ( $r$ 's .39-.62). In both conditions, more speed variability was marginally significantly associated with worse processing speed. No significant correlations emerged between neurocognitive variables and speed or braking reaction time.

Table 3. Simulated driving performance group differences

\begin{tabular}{|c|c|c|c|c|c|}
\hline \multirow[t]{2}{*}{ Variable } & \multicolumn{2}{|c|}{ Drivers with mTBI } & \multicolumn{2}{|c|}{ Controls } & \multirow[b]{2}{*}{$\underline{\mathrm{t}}$} \\
\hline & Mean (SD) & $\underline{\text { Range }}$ & Mean (SD) & $\underline{\text { Range }}$ & \\
\hline \multicolumn{6}{|l|}{ No Load - No Hazard } \\
\hline Average driving speed (mph) & $65.90(4.97)$ & $51.94-73.38$ & $67.67(4.96)$ & $60.10-79.55$ & 1.14 \\
\hline Speed variability $(\mathrm{mph})$ & $3.58(2.53)$ & $1.13-11.36$ & $3.42(2.60)$ & $0.93-8.64$ & -0.2 \\
\hline Standard deviation of lane position (ft) & $0.86(0.31)$ & $0.44-1.73$ & $0.88(0.32)$ & $0.37-1.52$ & 0.17 \\
\hline \multicolumn{6}{|l|}{ No Load-Event Present } \\
\hline Average driving speed (mph) & $65.45(4.57)$ & $52.79-73.83$ & $66.44(5.36)$ & $55.79-79.08$ & 0.64 \\
\hline Speed variability $(\mathrm{mph})$ & $4.86(2.93)$ & $1.20-13.15$ & $5.79(4.64)$ & $1.85-20.40$ & 0.78 \\
\hline Standard deviation of lane position (ft) & $0.87(0.30)$ & $0.43-1.71$ & $0.91(0.40)$ & $0.35-1.99$ & 0.36 \\
\hline Braking reaction time $(\mathrm{s})$ & $1.44(0.42)$ & $0.83-2.23$ & $1.46(0.36)$ & $0.72-1.92$ & 0.1 \\
\hline \multicolumn{6}{|l|}{ Concurrent Load - No Hazard } \\
\hline Average driving speed (mph) & $67.57(5.22)$ & $53.90-75.89$ & $68.31(7.50)$ & $59.16-85.39$ & 0.37 \\
\hline Speed variability $(\mathrm{mph})$ & $3.87(1.95)$ & $1.51-8.71$ & $3.59(1.97)$ & $1.07-7.25$ & -0.45 \\
\hline Standard deviation of lane position (ft) & $0.83(0.32)$ & $0.31-1.54$ & $0.75(0.22)$ & $0.38-1.21$ & -1.01 \\
\hline \multicolumn{6}{|l|}{ Concurrent Load - Event Present } \\
\hline Average driving speed (mph) & $66.16(5.56)$ & $51.95-74.04$ & $65.70(7.78)$ & $48.10-88.82$ & -0.22 \\
\hline Speed variability $(\mathrm{mph})$ & $5.45(2.93)$ & $2.41-15.55$ & $6.32(2.97)$ & $1.77-11.34$ & 0.94 \\
\hline Standard deviation of lane position (ft) & $0.74(0.21)$ & $0.36-1.09$ & $0.75(0.28)$ & $0.29-1.52$ & 0.1 \\
\hline Braking reaction time $(\mathrm{s})$ & $1.55(0.31)$ & $1.04-2.05$ & $1.58(0.17)$ & $1.30-1.85$ & 0.29 \\
\hline
\end{tabular}

Table 4. Driving performance differences in cognitive load conditions by group

\begin{tabular}{|c|c|c|c|c|c|}
\hline \multirow[t]{2}{*}{ Driving Performance Variable } & \multicolumn{2}{|c|}{ No Cognitive Load } & \multicolumn{2}{|c|}{ Cognitive Load } & \multirow[t]{2}{*}{$t$} \\
\hline & Mean (SD) & $\underline{\text { Range }}$ & Mean (SD) & Range & \\
\hline Drivers with $m T B I$ & & & & & \\
\hline Average driving speed & $65.90(4.97)$ & $51.94-73.38$ & $67.57(5.22)$ & $53.90-75.89$ & 2.19 \\
\hline Speed variability & $3.58(2.53)$ & $1.13-11.36$ & $3.87(1.95)$ & $1.51-8.71$ & 0.42 \\
\hline Standard deviation of lane position & $0.86(0.31)$ & $0.44-1.73$ & $0.83(0.32)$ & $0.31-1.54$ & -0.88 \\
\hline Controls & & & & & \\
\hline Average driving speed & $67.67(4.96)$ & $60.10-79.55$ & $68.31(7.50)$ & $59.16-85.39$ & 0.75 \\
\hline Speed variability & $3.42(2.60)$ & $0.93-8.64$ & $3.59(1.97)$ & $1.07-7.25$ & 0.47 \\
\hline Standard deviation of lane position & $0.88(0.32)$ & $0.37-1.52$ & $0.75(0.22)$ & $0.38-1.21$ & -3.18 \\
\hline
\end{tabular}

Note. Bold indicates $p<.05$.

\section{CONCLUSION}

The present pilot study examined young drivers acutely post-mTBI and is among the first to assess driving performance immediately after mTBI using a high-fidelity driving simulator. A simulated environment enabled us to perform a controlled experiment and expose drivers to risky driving conditions without physical risk. Hypotheses were partially supported. No significant difference between groups on any of the driving performance outcomes was observed. This pilot 
study's small sample may have limited statistical power to detect group differences or the simulator scenario may have lacked the sensitivity to detect subtle group differences as it featured mostly a straight roadway geometry to minimize potential simulator sickness. While our study suggested it is safe and feasible to enroll participants acutely post-injury, it would be important in the future to consider simulator scenarios that are increasingly complex and possibly more sensitive to detecting driving performance (e.g., driving performance of concussed individuals around curves, see Schmidt et al., 2016) in this at-risk, clinical population.

This study is among the first to quantify the impact of cognitive load on driving performance among young drivers with and without mTBI. We included two secondary tasks: talking with a passenger, one of the most prevalent tasks among young drivers (Gershon, Zhu, Klauer, Dingus, \& Simons-Morton, 2017), and an auditory operation span task (e.g., mental math while recalling words from memory). Both tasks suppress brain activity in areas needed for driving, slow reaction time, and result in missed cues by healthy drivers (Strayer et al., 2013). Individuals with mTBI drove significantly faster in the high cognitive load condition. This finding was surprising given the extensive distracted driving literature suggesting typically developing (healthy) drivers tend to slow their speeds under such conditions. Controls showed significantly less lane position variability in the high cognitive load condition. Lane variability findings are consistent with several studies showing reduced lane variability with increased cognitive load in uncomplicated driving conditions (Neyens et al., 2015), such as that performed here. No statistical difference was found in lane position variability between the cognitive load conditions in the mTBI group.

Driving metrics were significantly associated with various neurocognitive domains, supporting our hypotheses. Deficits in neurocognitive processing speed after mTBI may negatively affect driving performance (Ratcliff \& McKoon, 2008). Our findings suggest neurocognitive targets should be considered in future intervention development to accelerate the return to drive for young individuals with mTBI.

\section{ACKNOWLEDGEMENTS}

This work was supported by funding from the UAB CAS Interdisciplinary Innovation Team Grant Award and the OSU Chronic Brain Injury: Discovery Theme Initiative Award. Special thanks to the TRIP Lab, OSU Driving Simulation Lab, and Nationwide Children Hospital $(\mathrm{NCH})$, and for physicians at Children's of Alabama (COA)/ UAB, OSU, and $\mathrm{NCH}$ Concussion Clinics for providing access to patients. Thanks to the UAB Edward R. Roybal Center for Translational Research in Aging and Mobility (NIH/NIA Grant 5 P30 AG022838-09) for providing facilities/resources. Finally, thank you to Dr. Andrea Underhill for the critical review.

\section{REFERENCES}

Baker, A., Unsworth, C. A., \& Lannin, N. A. (2015). Determining fitness to drive: A systematic review of the methods and assessments used after mild traumatic brain injury. Br J Occup Ther, 78(2), 73-84.

Centers for Disease Control and Prevention. (2016). TBI: Get the facts. Retrieved from http://www.cdc.gov/traumaticbraininjury/get_the_facts.html

Collie, A., Darby, D., \& Maruff, P. (2001). Computerised cognitive assessment of athletes with sports related head injury. Br J Sports Med, 35(5), 297-302. 
Cossette, I., Ouellet, M. C., \& McFadyen, B. J. (2014). A preliminary study to identify locomotor-cognitive dual tasks that reveal persistent executive dysfunction after mild traumatic brain injury. Arch Phys Med Rehabil, 95(8), 1594-1597.

Del Rossi, G. (2017). Evaluating the Recovery Curve for Clinically Assessed Reaction Time After Concussion. J Athl Train, 52(8), 766-770.

Gershon, P., Zhu, C., Klauer, S. G., Dingus, T., \& Simons-Morton, B. (2017). Teens' distracted driving behavior: Prevalence and predictors. J Safety Res, 63, 157-161.

Grady, M. F., Master, C. L., \& Gioia, G. A. (2012). Concussion pathophysiology: rationale for physical and cognitive rest. Pediatr Ann, 41(9), 377-382.

Insurance Institute for Highway Safety [IIHS]. (2013). Fatality facts: Teenagers 2013. Retrieved from http://www.iihs.org/iihs/topics/t/teenagers/fatalityfacts/teenagers/2013

Marshall, S., Bayley, M., McCullagh, S., Velikonja, D., \& Berrigan, L. (2012). Clinical practice guidelines for mild truamatic brain injury and persistent symptoms. Can Fam Physician, $58(3), 257-267$.

McCartt, A. T., Shabanova, V. I., \& Leaf, W. A. (2003). Driving experience, crashes and traffic citations of teenage beginning drivers. Accid Anal Prev, 35(3), 311-320.

McCrory, P., Meeuwisse, W. H., Aubry, M., Cantu, R. C., Dvorak, J., Echemendia, R. J.,... Turner, M. (2013). Consensus statement on concussion in sport: the 4th International Conference on Concussion in Sport, Zurich, November 2012. J Athl Train, 48(4), 554-575.

McKinlay, A., Grace, R. C., Horwood, L. J., Fergusson, D. M., Ridder, E. M., \& MacFarlane, M. R. (2008). Prevalence of traumatic brain injury among children, adolescents and young adults: Prospective evidence from a birth cohort. Brain Inj, 22(2), 175-181.

Neyens, D. M., Boyle, L. N., \& Schultheis, M. T. (2015). The effects of driver distraction for individuals with traumatic brain injuries. Hum Factors, 57(8), 1472-1488.

Preece, M. H. W., Geffen, G. M., \& Horswill, M. S. (2013). Return-to-driving expectations following mild traumatic brain injury. Brain Inj, 27(1), 83-91.

Preece, M. H. W., Horswill, M. S., \& Geffen, G. M. (2010). Driving after concussion: The acute effect of mild traumatic brain injury on drivers' hazard perception. Neuropsychology, 24(4), 493-503.

Ratcliff, R., \& McKoon, G. (2008). The diffusion decision model: theory and data for two-choice decision tasks. Neural Comput, 20(4), 873-922.

Schmidt, J., Hoffman, N.L., Ranchet, M., Miller, S., \& Tomporowski, P. (2016). Driving after concussion: Is it safe to drive after symptoms resolve? J Neurotrauma, 34, 1571-1578.

Stavrinos, D., Jones, J. L., Garner, A. A., Griffin, R., Franklin, C. A., Ball, D., . . Fine, P. R. (2013). Impact of distracted driving on safety and traffic flow. Accid Anal Prev, 61, 63-70.

Strayer, D. L., Cooper, J. M., Turrill, J., Coleman, J., Medeiros-Ward, N., \& Biondi, F. (2013). Measuring cognitive distraction in the automobile. Retrieved from Washington, DC: https://aaafoundation.org/wp-content/ uploads/2018/01/MeasuringCognitiveDistractions Report.pdf

Strayer, D. L., Turrill, J., Cooper, J. M., Coleman, J. R., Medeiros-Ward, N., \& Biondi, F. (2015). Assessing Cognitive Distraction in the Automobile. Hum Factors, 57(8), 1300-1324.

Williams, A. F. (2003). Teenage drivers: Patterns of risk. J Safety Res, 34(1), 5-15. 